\title{
MicroRNA Expression Profiling by Bead Array Technology in Human Tumor Cell Lines Treated with Interferon-Alpha-2a
}

\section{Fredy Siegrist, Thomas Singer, and Ulrich Certa}

\begin{abstract}
MicroRNAs are positive and negative regulators of eukaryotic gene expression that modulate transcript abundance by specific binding to sequence motifs located prevalently in the $3^{\prime}$ untranslated regions of target messenger RNAs (mRNA). Interferon-alpha-2a (IFN $\alpha$ ) induces a large set of protein coding genes mediating antiproliferative and antiviral responses. Here we use a global microarray-based microRNA detection platform to identify genes that are induced by IFN $\alpha$ in hepatoma- or melanoma-derived human tumor cell lines. Despite the enormous differences in expression levels between these models, we were able to identify microRNAs that are upregulated by IFN $\alpha$ in both lines suggesting the possibility that interferon-regulated microRNAs are involved in the transcriptional repression of mRNA relevant to cytokine responses.
\end{abstract}

Key words: MicroRNAs, Oligonucleotide Array Sequence Analysis, Interferons, Melanoma, Hepatoma, Reverse Transcriptase Polymerase Chain Reaction, Suppressor of Cytokine Signaling Proteins.

\section{Introduction}

The gene expression patterns of tumor-derived cell lines differ greatly, as do their responses to antiproliferative effects of interferons (IFNs). The cause of this variation has been under investigation for more than 40 years, but only basic regulatory mechanisms of interferon signaling are understood today. Small regulatory genome encoded RNAs, such as microRNAs, have recently attracted attention in genomic research. New methods to analyze the levels of these regulatory elements are now commercially available, but the power of these techniques is still discussed extensively. Our study was

Shulin Li (ed.), Biological Procedures Online, Volume 11, Number 1

(C) to the author(s) 2009

DOI: 10.1007/s12575-009-9012-1 URL: springerprotocols.com; springerlink.com 
designed to compare two methods for microRNA detection with respect to usefulness in defined cell culture assays. The experimental design assesses variation between the two cell lines and the treatment effects of IFN $\alpha$.

A hallmark of the therapeutic activity of type I interferons is the induction of antiproliferative activity mediated by the upregulation of several hundred response genes with pleiotropic functions (1). These genes can be divided into two major classes based on the kinetic properties of induction (2). Primary response genes (PRGs) are upregulated within $24 \mathrm{~h}$ after the cytokine signal and the secondary response genes (SRGs) are induced following day 1 when the activity of the PRGs decays. In contrast to SRGs, all PRGs studied to date contain bona fide interferon response elements in the promoter region, which are required for binding of the interferon-stimulated gene factor 3 (ISGF3) complex and for janus kinase/signal transducer of transcription (JAK/STAT)pathway-mediated signaling.

Expression of PRGs is turned off by proteins termed suppressors of cytokine signaling (SOCS) (3). As the nomenclature indicates, this class of polypeptides has the capacity to interfere and silence other cytokine-induced signaling cascades (for review see (4)). SOCSI for instance is part of the early inducible PRG cluster and down modulation occurs together with the other genes before onset of SRG expression. It is believed that feedback inhibition of JAK/ STAT signaling by SOCSI represses transcriptome modulation of IFN $\alpha$ signaling (5). Regulation of SOCS protein translation by interferon-regulated microRNAs (IRmiRs) would enhance the potential of cytokine fine regulation. It has been reported that miR-19 antagonists lead to higher SOCS1 levels and miR-19 mimics can repress SOCSI reporter constructs, thus obviously supporting the bioinformatic predictions that SOCSI is a direct target of miR-19 (6). Inhibition of SOCS activity could for instance prolong the duration of cytokine activity, which has obvious clinical implications.

Following the discovery of microRNAs in virtually all higher eukaryotic organisms significant research efforts were initiated to address the function of these catalytic oligonucleotides which are the natural counterparts of synthetic small inhibitory RNAs (siRNAs) used for experimental gene silencing (for review see (7)). MicroRNAs are positive and negative modulators of the expression of entire gene clusters that contain complementary microRNA recognition sequence motifs in the $3^{\prime}$-UTR. Today, prediction of microRNA target genes by homology-based algorithms is still ambiguous (8). The activity of one or several microRNAs could explain suppression of the entire PRG cluster provided that microRNA abundance is regulated by IFN $\alpha$. Alternatively, microRNA-mediated degradation of transcripts encoding negative regulatory proteins would also abolish PRG expression and restore IFN $\alpha$ responsiveness. 
Some recent reports showed that interferon beta (IFN $\beta$ ) stimulation can boost microRNA levels in cell culture together with inhibition of viral replication (9). At this point it is an open question whether this induction is IFN $\beta$ specific or a shared feature of all type I interferons. To investigate whether microRNA are also involved in regulation of IFN $\alpha$ response, we used two human-tumor-derived cell lines: the melanoma line ME-15 (10) and the hepatoma line HuH7 (11). We have chosen these cell lines as models, because we have a good understanding of the IFN $\alpha$ responses at the mRNA and the protein levels in these cell lines. Further we chose to use a melanoma cell line because IFN is also used for treatment of this cancer type. HuH7 is commonly used as a model for testing antiviral effects of IFN in the HCV replicon system. In both models efficient responses to IFN $\alpha$ have been shown at the functional and transcriptional level. IFN $\alpha$ response genes carry response elements in their promoter region and these motifs are responsible for gene expression with similar efficiency in many cell types. Therefore we expected to find a similar regulated set of genes in both lines given that IRmiR genes are regulated by the same mechanism, whereas some constitutively expressed microRNA genes were expected to be cell type specific for functional reasons. We have chosen a DNA-microarray-based technology (Illumina) for the multi-parallel expression analysis of all known human microRNAs (http://microrna.sanger.ac.uk/; Release 10.0: August 2007). This method allowed us to process total RNA as template, allowing the possibility of mRNA gene expression profiling in further experiments. Briefly, annealing of microRNA specific primers combined with enzymatic polyadenylation allows multi-parallel polymerase chain reaction (PCR)-mediated amplification of individual microRNAs. The output of this step is a DNA amplicon library that reflects to a large extent the original stoichiometry of mature microRNAs in a cell or tissue (12). PCR amplification is performed with fluorescently labeled primers, which allows quantitative signal detection by conventional confocal laser scanning.

\section{Materials and Methods}

2.1. Cell Culture, Interferon Treatment, and RNA Precipitation
Melanoma cells (ME-15) were cultured in RPMI 1640 with L-Glutamine supplemented with non-essential amino acids and sodium pyruvate $(1 \mathrm{mM})$ and hepatoma $(\mathrm{HuH}$ ) cells were cultured in DMEM + GlutaMAX. Both media contained 10\% FBS. All cell culture reagents were purchased from Invitrogen 


\subsection{Illumina Bead Array MicroRNA Detection}

\subsection{Data Processing and Statistical Analysis}

\subsection{Quantitative PCR and Data Processing}

(GIBCO $\left.{ }^{\circledR}\right)$. Roferon (Interferon alpha2a, ROCHE) was diluted in fresh medium to a final concentration of $1,000 \mathrm{U} / \mathrm{mL}$ and control cultures were grown without cytokine. Cells were cultured at $37^{\circ} \mathrm{C}$ in a humidified atmosphere containing $5 \% \mathrm{CO}_{2}$. Total RNA preparation was carried out using TRIZOL (Invitrogen) total RNA extraction using $1 / 2$ volume of 1 -bromo-3-chloro-propane (molecular biology grade, SIGMA) as chloroform substitute. For efficient recovery of small RNAs, DNA LoBind tubes (Eppendorf) were used and all centrifugation steps were performed at maximum speed and $4^{\circ} \mathrm{C}$ in an Eppendorf 5417R centrifuge. Total RNA was precipitated with $2 \mathrm{vol}$ of 2 -propanol (Fluka) at $-20^{\circ} \mathrm{C}$ for at least $16 \mathrm{~h}$. The RNA pellet was washed with $75 \%$ ethanol (Merck), dried, and dissolved in DEPC-treated water (Ambion). The RNA was quantified with Quant-iT ${ }^{\mathrm{TM}}$ RiboGreen ${ }^{\circledR}$ RNA Assay (Invitrogen) as suggested by Illumina.

Starting with $500 \mathrm{ng} /$ sample of total RNA, mature microRNAs were amplified with the Illumina human vl MicroRNA expression profiling kit containing primers for 743 human microRNAs. The resulting amplicons were hybridized to a 96 sample universal probe capture array and fluorescent signals were detected by confocal laser scanning. All steps were performed according to Illumina's instructions manual.

The data was processed with Beadstudio software (version 3.1.3, gene expression module 3.3.8) including the calculation of detection $p$ values based on negative control bead signals. Log-transformation, loess normalization (13) and statistical analysis were performed with $\mathrm{R}$ (2.8.1) (14) using the package lumi (1.8.3) (15) and software contained therein, in particular limma (2.16.4) (16). Statistical models were chosen as follows: a linear model (limma $t$ statistics) with two separate coefficients for HuH7 and ME-15 cells was used for the selection of differently expressed genes shown in Fig. 1 and Table 1. Statistics represented in the tables were calculated by testing the two indicated conditions as independent factors. In Table $1, p$ values were adjusted by the false discovery rate method (17). Treatment effects shown in Table $2 \mathrm{~b}$ and Fig. 3 a were modeled with two coefficients (cell line, treatment) for time point $4 \mathrm{~h}, p$ values arise from $t$ statistics. Normalized relative fluorescence levels were calculated by $2^{\wedge}$ mean (of $\log 2$ transformed, loess normalized values). Change factors (CHF) were calculated as fold change on the linear scale minus $\mathrm{l}$ as previously described (2). Raw data, non-normalized, and normalized microRNA expression data have been submitted to the Gene Expression Omnibus with accession number GSE16421.

microRNA levels were measured using TaqMan ${ }^{\circledR}$ microRNA assays (Applied Biosystems) using the TaqMan ${ }^{\circledR}$ MicroRNA reverse transcription (RT) kit with TaqMan ${ }^{\circledR} 2 \times$ universal PCR master 


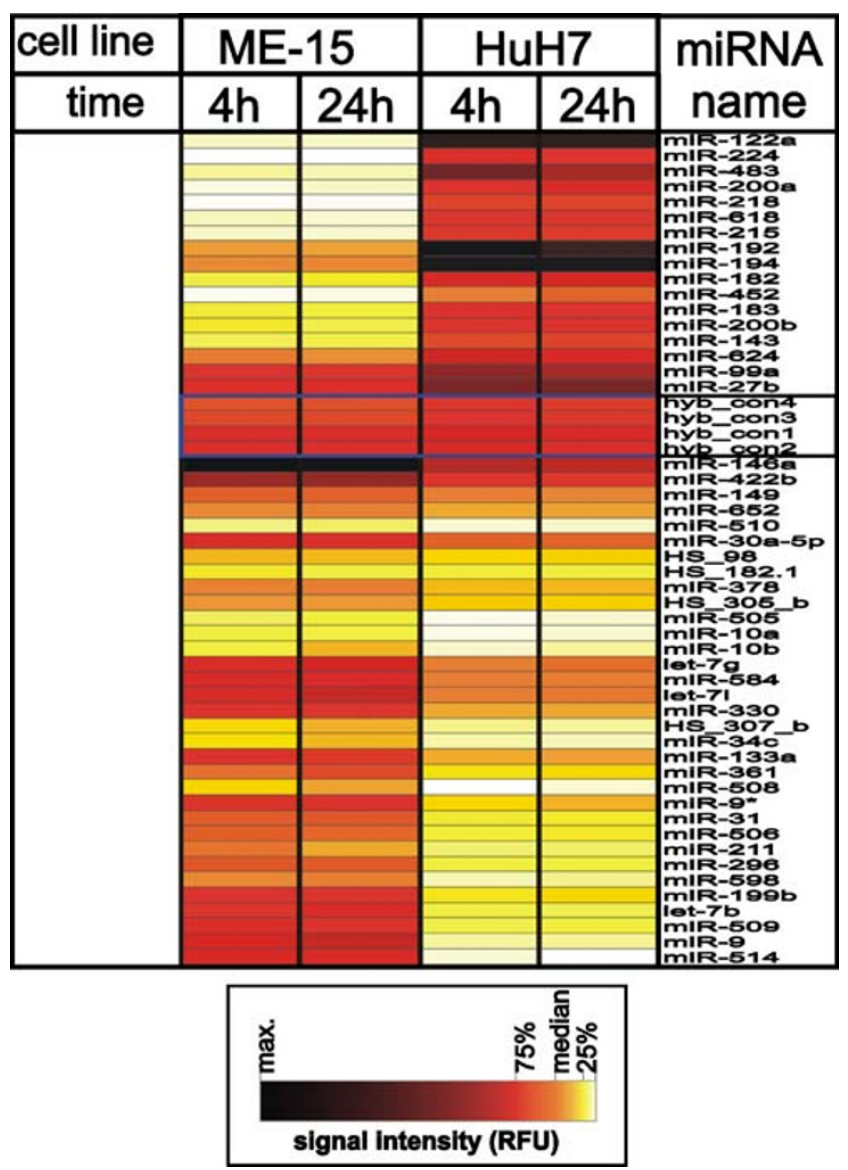

Fig. 1. Differential microRNA expression in human melanoma (ME-15) and hepatoma $(\mathrm{HuH} 7)$ cells. microRNA expression levels were compared in two cell lines at two different time points and corrected for the treatment effect. The 50 most significant ( $p$ value below $10^{-12}$ ) microRNA expression values from untreated samples are shown in a heat diagram including hybridization controls as reference for technical variance. White indicates noise levels, yellow indicates the first quartile, orange the median, red the third quartile, and black maximum expression levels. The intensity data, significance values and the IFNa-dependent expression levels are summarized in Table 1.

mix (No AmpErase® UNG) as recommended by the supplier. Ten nanograms of total RNA was used as input for amplification using the samples used for microarray analysis. Reversed transcriptase products were diluted 1:15 and measured on an ABI 7900HT fast real-time PCR system. Technical replicates were run on three different plates (one with 40 cycles and two with 50 cycles) and threshold for cycling time (CT) calculation was set for all probes to 0.2 . For estimation of endogenous small RNA content, the nucleolar RNA RNU48 was used as control and reference. 


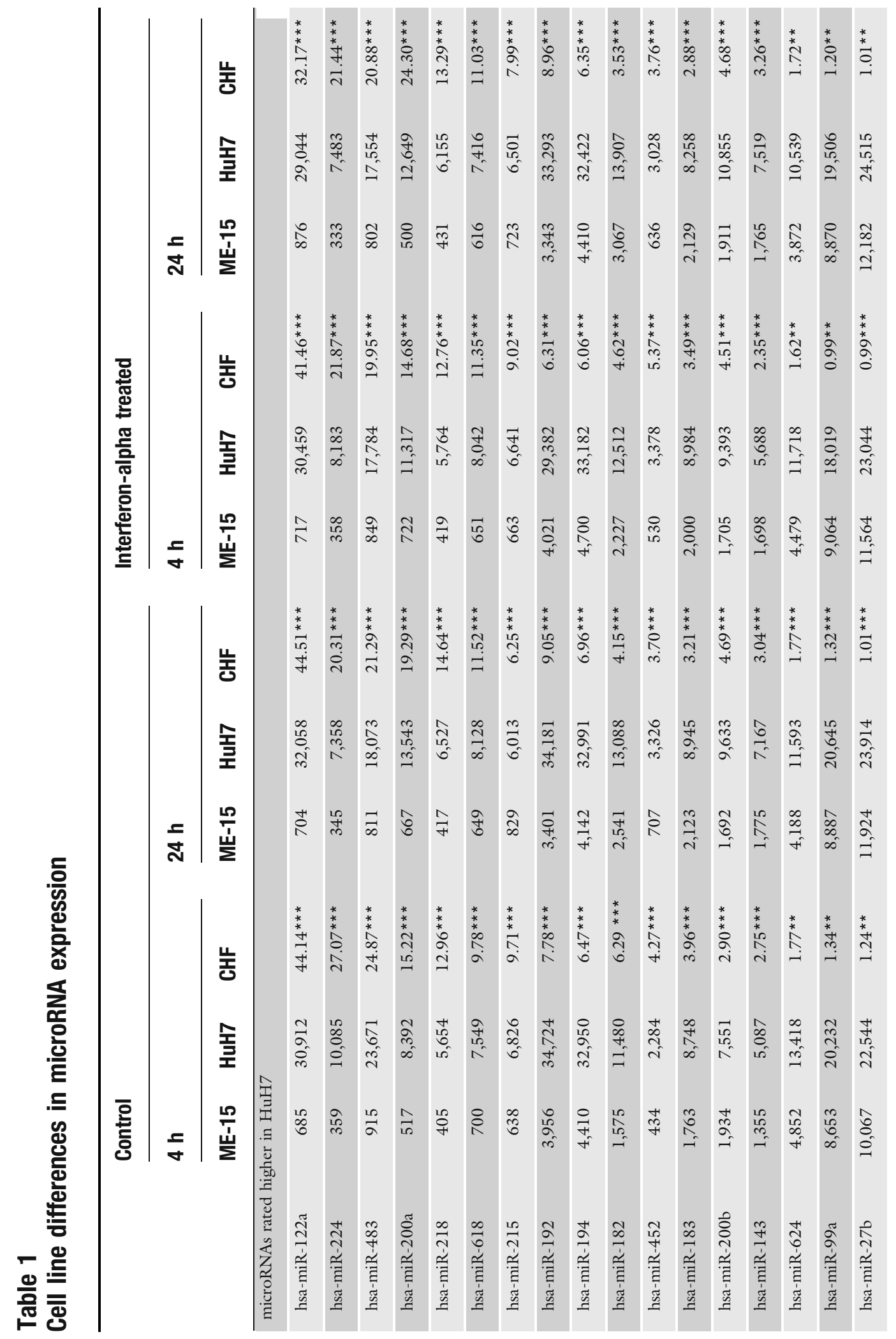




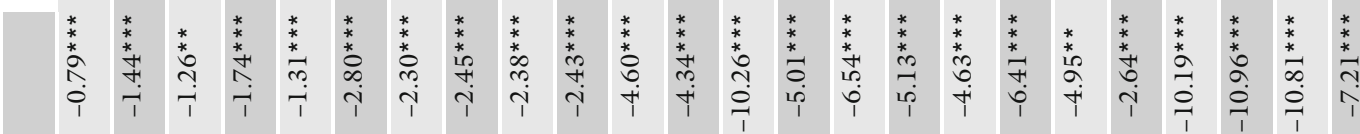

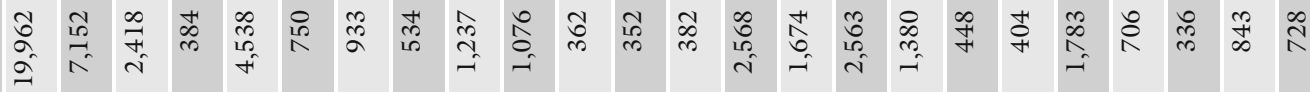

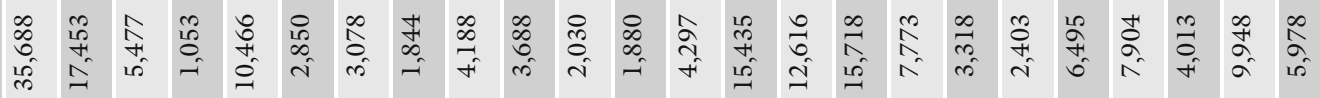

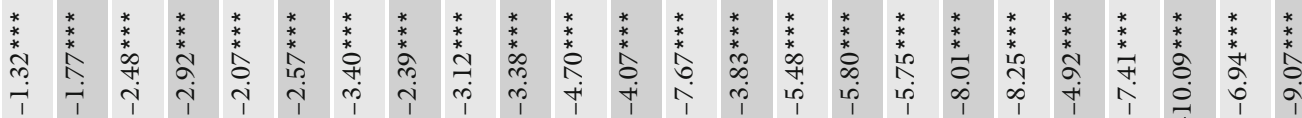

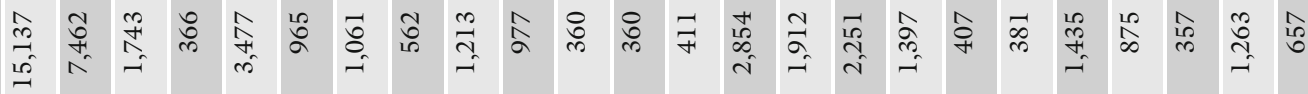

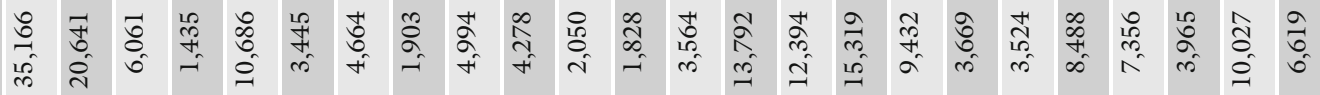

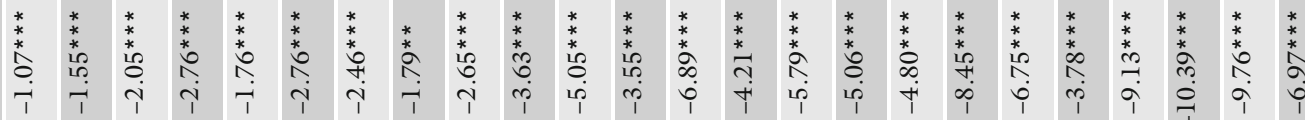

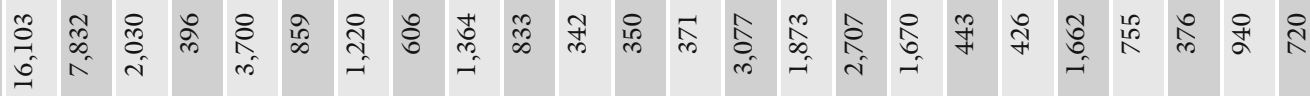

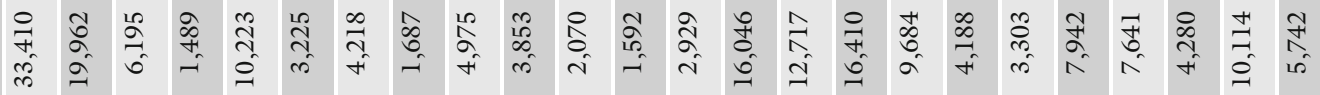

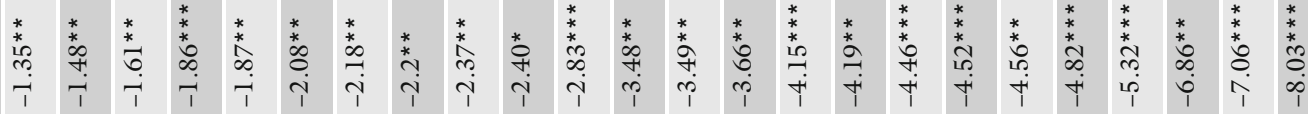

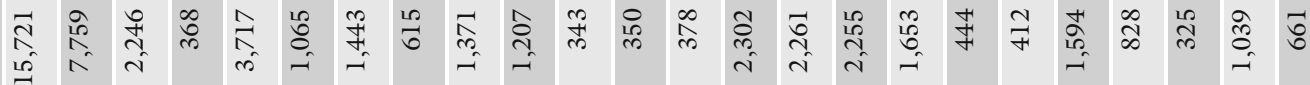

$\stackrel{10}{-19}$

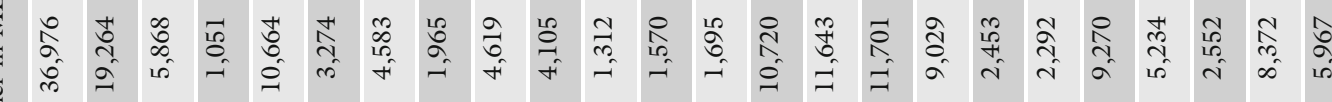

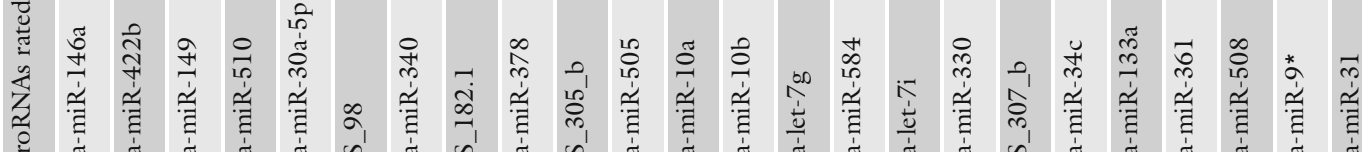

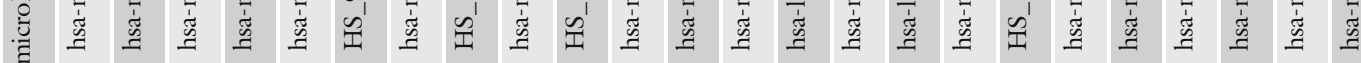




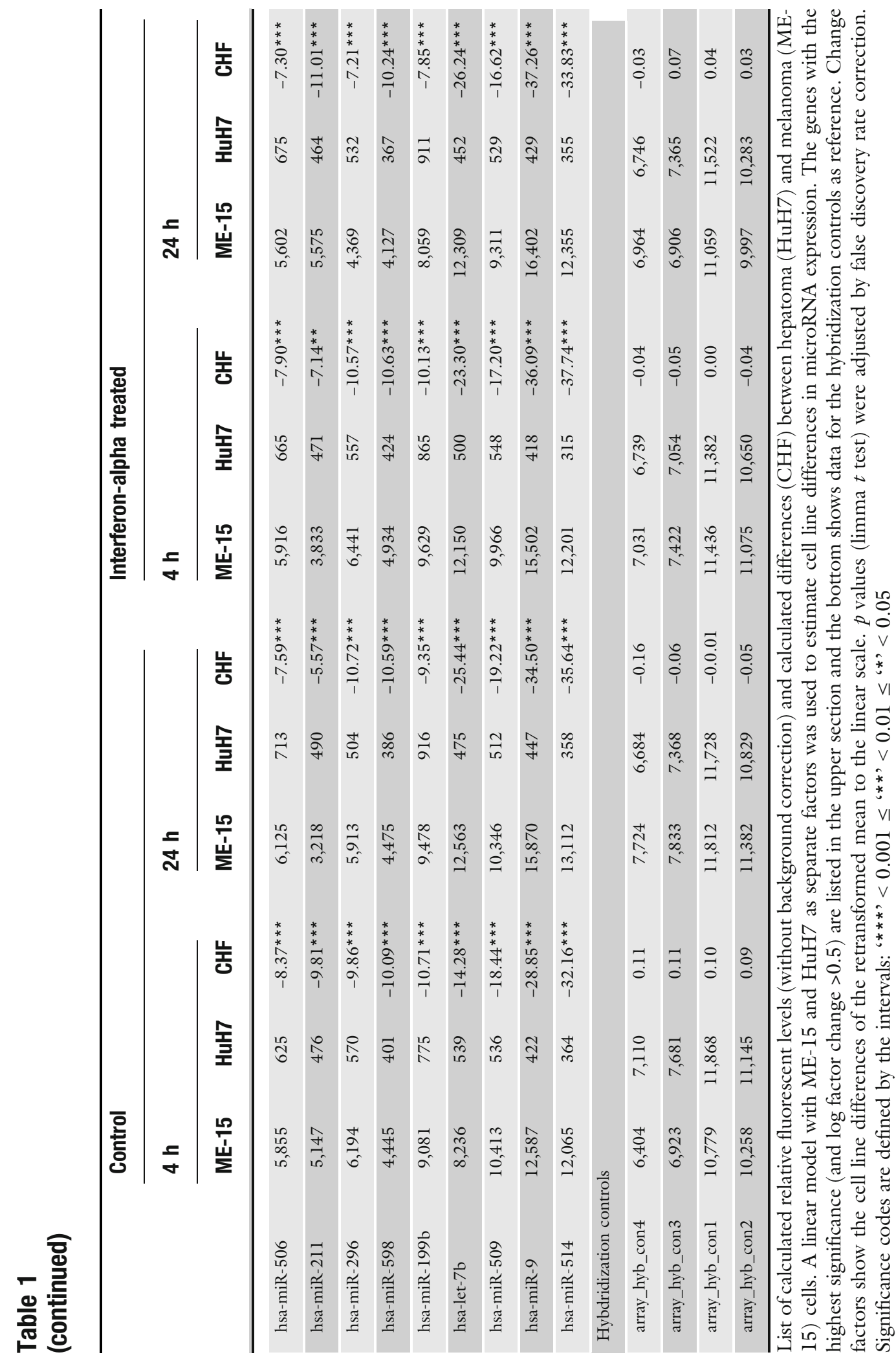




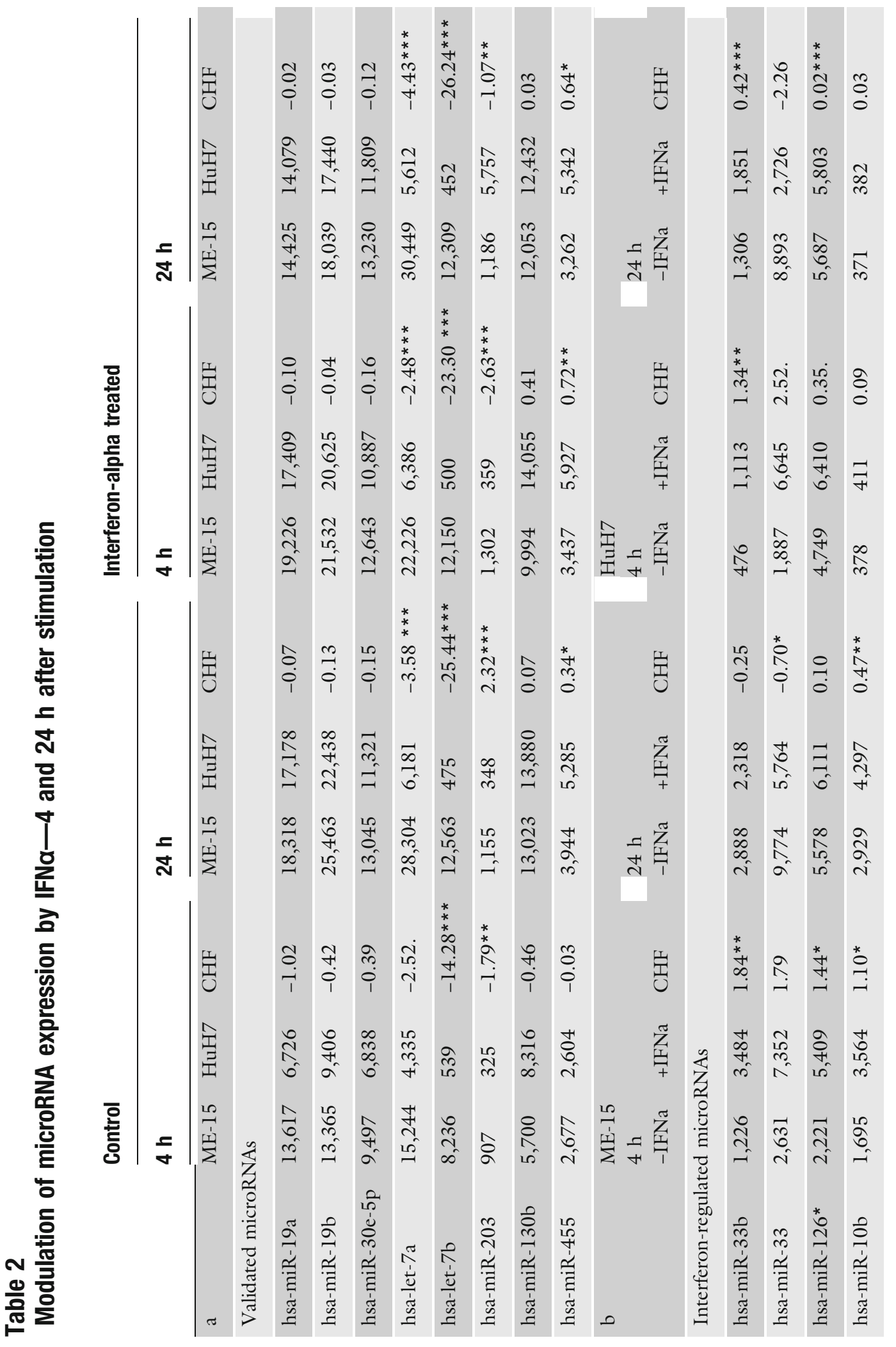




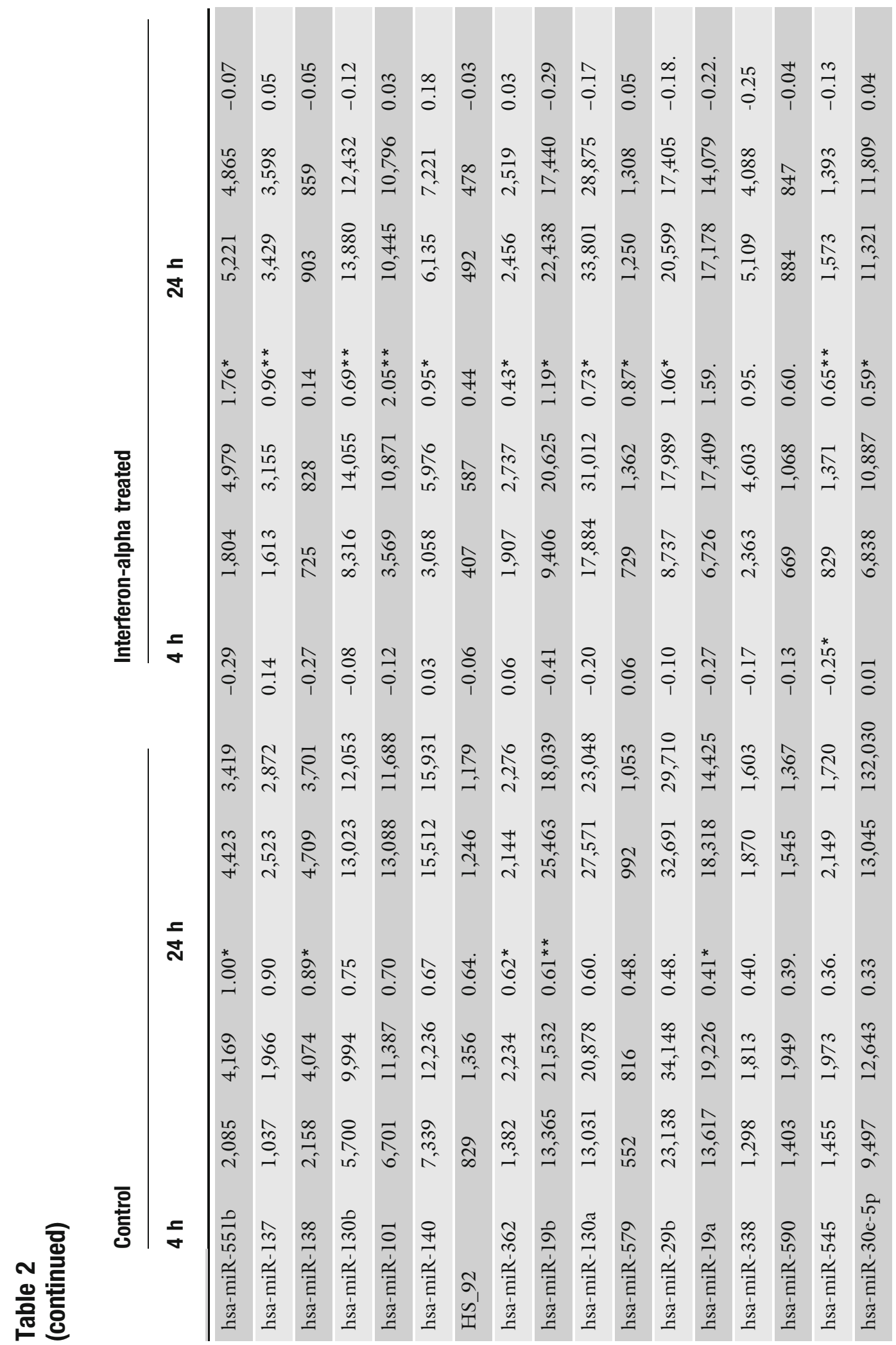




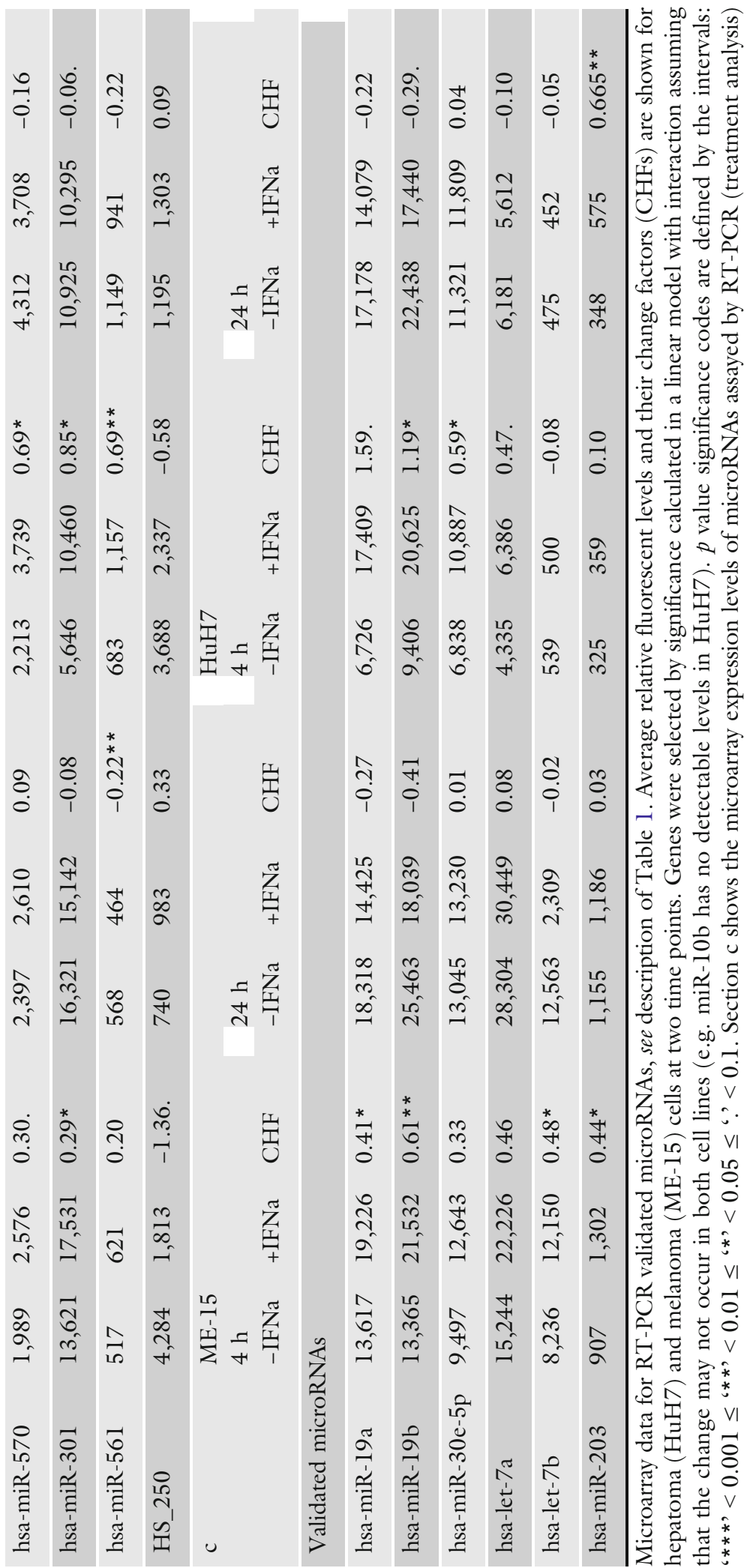




\section{Results and Discussion}

The following technical aspects have to be considered for result interpretation. The dataset of the microRNA bead array assay is not directly comparable to gene expression arrays where in vitro translated transcripts are directly hybridized to the probes. Moreover Illumina's bead array technology tends to have higher background fluorescence levels and lower change factor values than Genechips from Affymetrix. Background (average of negative control signals) and noise (standard deviation of negative probes from each sample) were $528 \pm 60$ and $229 \pm 67$, respectively. The density of all samples shows a bimodal distribution peaking around the background fluorescent levels and the robust levels (approximately 12,000). The curve is skewed to the right and peak density height is found in the ratio $4: 1$ considering all probes (data not shown). The distribution of probes detected in all samples (detection $p$ value

a

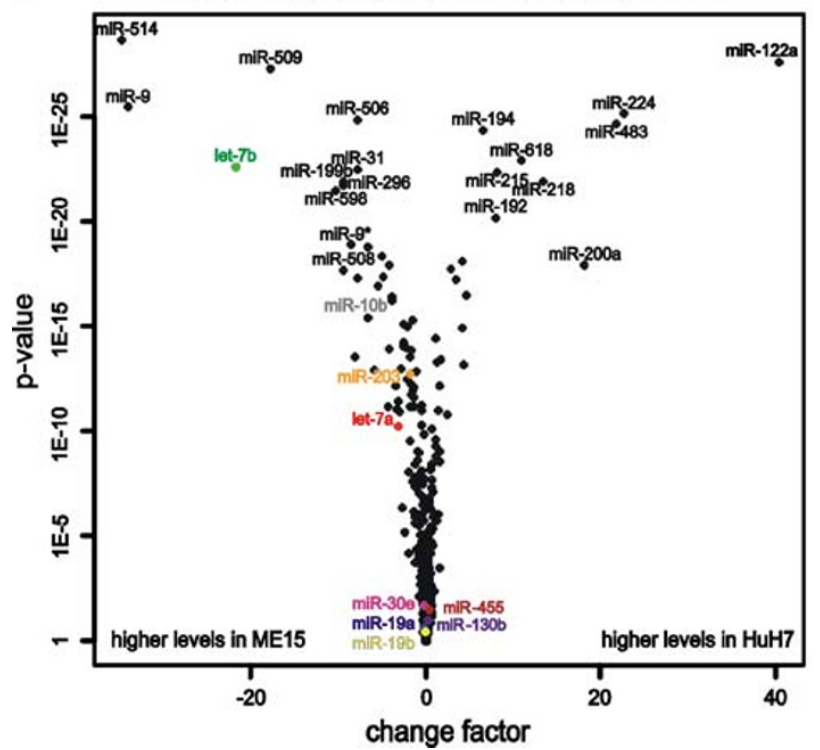

b MicroRNA levels messured with qPCR
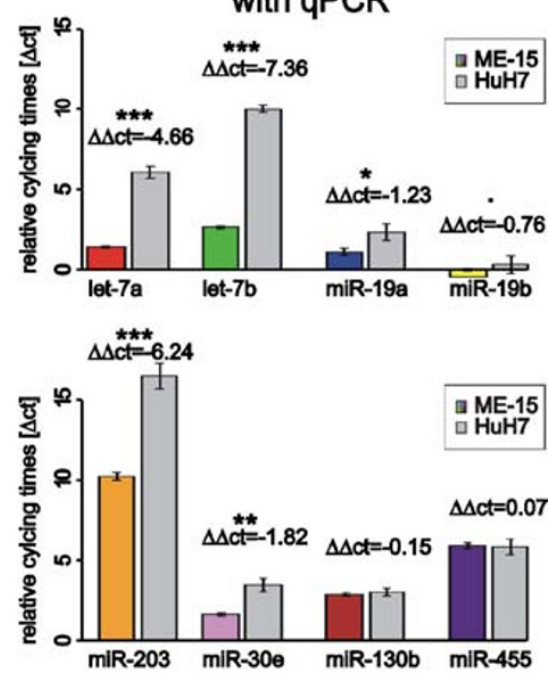

Fig. 2. Cell line specific microRNA expression Volcano plot (a) display demonstrates the multi-variant biological diversity of microRNA expression in ME-15 or HuH7 cells. The estimated fold-change value (change factor) is plotted on the $X$-axis against the $p$ value (limma $t$ statistics) in logarithmic scale on the $Y$-axis. A linear model, using the expression values of untreated ME-15 cells at $4 \mathrm{~h}$ as base together with three parameters to estimate differences in time, treatment, or cell line. The top ranked and GPCR measured microRNAs are annotated. $\mathbf{b}$ Quantitative PCR validation of microarray data using eight selected microRNAs. Input total RNA came from independent cell cultures. Data are shown as relative cycling times $(\triangle C T)$ calculated with endogenous control RNU18 for ME-15 (color-filled bars) and $\mathrm{HuH} 7$ (gray). Error bars represent $\Delta \mathrm{CT} \pm \Delta x$ (standard deviation of biological replicates). $\Delta \Delta \mathrm{CT}$ are noted above the bars together with the significance codes for $t$ statistics defined by the intervals ${ }^{\text {‘**' }}<0.001$ $\leq^{(* *)}<0.01 \leq{ }^{\text {(*) }}<0.05 \leq$ '.' $<0.1$. 
a

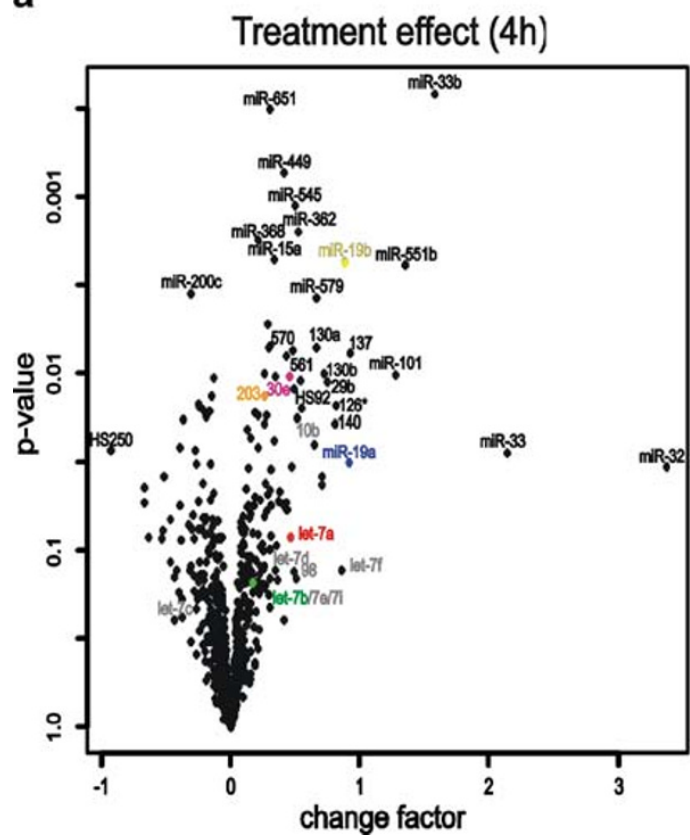

b mIR-19a miR-19b mIR-30e

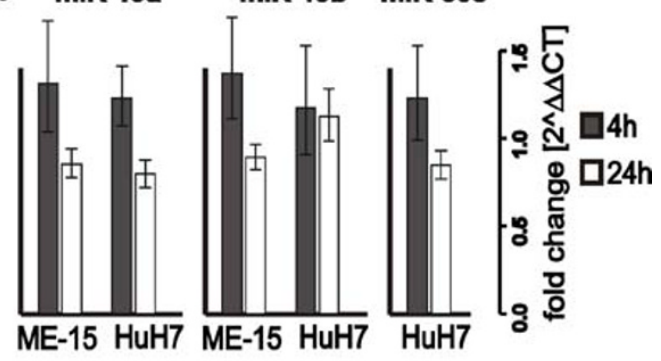

let-7a let-7b miR-203

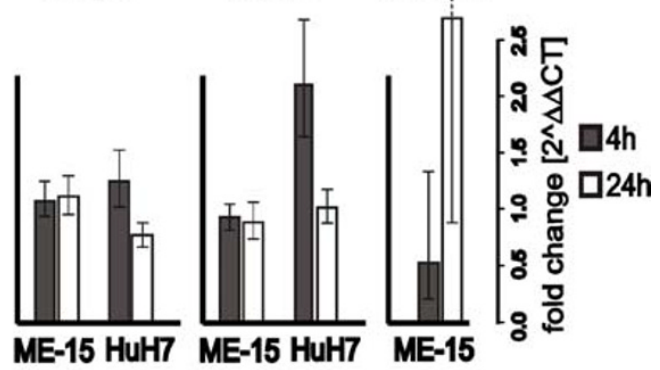

Fig. 3. IFNa-dependent modulation of microRNA expression. a Volcano plot display of IFNa induced microRNA upregulation $4 \mathrm{~h}$ after induction. The change factor values $\left(2^{\wedge} \log\right.$ factor change -1$)$ are plotted on the $X$-axis against the $p$ value in logarithmic scale on the $Y$-axis. Top-rated microRNAs are annotated together with the let-7 family members. b Confirmation of IFNa effect for selected microarray data by qPCR. The CT-values are the average of three technical and three biological replicates and changes were calculated with $2^{\wedge} \Delta \mathrm{MNE}$ (mean normalized expression values). Error bars show $2^{\wedge} \Delta \mathrm{MNE} \pm \Delta x$ (average standard error of treated and untreated $\mathrm{MNE}$ ) from biological triplicates. miR-30e failed to amplify in ME-15 and miR-203 was below the detection limit in $\mathrm{HuH7}$. Expression values were normalized against endogenous snoRNA RNU48 levels.

threshold at 0.01 ) has a plateau ranging from about 2,000 close to the detectors maximum capacity of $2^{\wedge} 16$ relative fluorescent units (12). As expected, the correlation of data coming from biological replicates $r^{2}=0.952 \pm 0.028$ (not normalized) and $r^{2}=0.956 \pm 0.022$ (after loess normalization and log-transformation) was lower than for technical replicates $r^{2}>0.97$ (12). We preferred loess normalization to quantile normalization because the later was too aggressive for the given small probe numbers.

As a first step we wished to address the robustness of the microRNA array in probe detection by selecting microRNA genes that are detected under all experimental conditions with high statistical significance in all biological triplicates (detection $p$ value $<0.01$ ). In each set of triplicate samples (control, $4 \mathrm{~h}$ or $24 \mathrm{~h}$, IFN $\alpha$ stimulation) we detect approximately 270 genes that fulfill the above criteria. This corresponds to roughly a third of microRNAs available for detection in the assay system. Furthermore, this result suggests indirectly that IFN $\alpha$ treatment does not induce global changes in microRNA gene expression, but it modulates rather the expression of individual genes. 
Today it is well established that microRNA expression patterns are cell and tissue type specific, which is consistent with a role in cell differentiation and biological function (8). Thus we expected to detect genes with preferential expression in either hepatoma or melanoma cells as these cell lines are derived from different tumors. Indeed, when all experimental conditions and data points are included in the data analysis about 150 microRNAs genes show preferential expression in either $\mathrm{HuH7}$ or ME- 15 cells (Fig. 1). Table 1 shows the expression data for the most significant genes including change factors and significance score as reference. Among these differentially expressed genes there are three members of the let-7 family, which has properties of tumor suppressor genes (for review see (18)). Therefore it is not surprising that the members of this well-known microRNA gene family are deregulated in the analyzed cancer cells too. Furthermore, the different developmental stage of our cancer cell lines is expected to have left a genomic fingerprint where some microRNA genes are expressed in one but not the other cell line (19). Consistent with this, expression of some microRNAs is strictly cell type specific and barely detectable in the other cell type (Fig. 2a), for example the liver-specific miR-122a and miR-192 (20).

Bead-array-based microRNA detection technology, including the bio-statistic analysis, is currently not well established or widely used and we have applied a commercial PCR-based assay to confirm the array data for some microRNAs that cover different expression levels and change factors. In contrast to mRNA profiling, where RT-PCR-based assays are considered as gold standard for data validation, new generation deep sequencing is considered as the method of choice for microRNA quantification but is not available in our research institute. For the microRNAs let-7 a/b, miR-19 a/b, and miR-203, the PCR-based quantification method (Fig. 2b) confirmed the direction of change found with microarray technology (Table 2a). Expression of miR-130b and miR-455 was at similar levels in both assays. The correlation calculated for the eight tested microRNAs was acceptable: multiple $r^{2}$ from $f$ test of mean relative cycling times $(\Delta \mathrm{CT})$ to mean $\log 2$ microarray expression values was 0.9279 . Differences of absolute levels between the microRNA targets probably results from different hybridization properties of the microarray probes and variation in the performance of Taqman primers for the specific microRNA on the other side.

Assuming that any IFN $\alpha$ relevant microRNA will have the same kinetics as the mRNA for PRGs, we looked at the regulation of microRNA genes in our experiment. These IRmiRs should respond to IFN $\alpha$ stimulation preferentially in both cell lines, because this would be a good indication of a general mechanism in the IFN $\alpha$ response. Within the 25 most significantly regulated genes (Table 2b), only one gene (HS_250) is downregulated. A 
general upregulation of transcripts is consistent with classic IFN $\alpha$ signaling seen for mRNAs. However, the maximal observed change factor with high significance was 1.84 ( $\mathrm{miR}-33 \mathrm{~b}$ in Table $2 \mathrm{~b}$ ) which is clearly lower than the values seen for protein coding mRNAs (2). We also included an expression analysis $24 \mathrm{~h}$ after IFN $\alpha$ stimulation in order to detect microRNA genes that show either delayed induction or remain activated at comparable levels to the $4 \mathrm{~h}$ stimulus. Based on our data set, the majority of the microRNA response genes show no further induction, but rather moderate downregulation $24 \mathrm{~h}$ after induction. This finding is not surprising as we expected immediate early impact of IFN $\alpha$-mediated primary signaling.

We also measured the IFN $\alpha$ response in the same experiment and for the same microRNAs (Table 2c). When we analyze the IFN $\alpha$ effect at the early time point in both cell lines we find all the validated microRNAs to be upregulated (Fig. 3a). The magnitude of upregulation and the basal expression levels of the microRNA-19a and 19b are similar in both cell lines (Fig. 3b, top). This and the finding that miR-19 regulates SOCS1 (4) may be relevant for the regulation of cytokine signaling. let-7a and let-7b had higher levels in the melanoma-cell-line-derived samples compared $\mathrm{HuH} 7$, but the induction by IFN $\alpha$ in ME- 15 could not be reproduced by RT-PCR (Fig. 3b, bottom). In both assays accurate fold changes are difficult to calculate, if the baseline expression level is close to background noise or the detection limit. An example of a gene at the detection limit is miR-203, which is not detectable without IFN $\alpha$ treatment in $\mathrm{HuH7}$ cells (Table 2c). Upon IFN $\alpha$ stimulation ( $24 \mathrm{~h}$ in $\mathrm{HuH} 7$ ) the microRNA is detectable above background suggesting minimal induction. Consequently a solid change factor cannot be calculated, which is consistent with the high variance obtained by qPCR (ME-15). This result is in fact not surprising, because both technologies rely on logarithmic PCR amplification of microRNA templates. At low expression levels, both technologies show relatively high variation in biological replicates, which should be considered for data interpretation. Interestingly, miR-203 has a putative binding site for ISGF3 in the promoter region, which would enable IFN $\alpha$-dependent upregulation. miR-30 has been reported to be IFN $\beta$ inducible, although the subclass measured was not specified by the authors (9). We decided to analyze the most promising candidate (miR-30e-5p) present in our microarray dataset (Fig. 3a in gray). Detection of miR-30e failed in ME- 15 cells due to technical problems, but induction in $\mathrm{HuH7}$ was similar to miR-19a/b.

Some technology-related questions remain open. The microRNA assay measures essentially the number of amplicons generated by RT-PCR for each transcript. Thus the signal is an indirect measurement of transcript abundance as compared to classical mRNA microarray platforms, where the target mRNA is directly 
labeled during linear amplification by in vitro transcription. As a consequence, change factor calculations for amplicon-based assays are ambiguous.

In summary, Illumina's bead array technology is well suited for multi-parallel profiling of microRNAs expressed in different cell types or tissues. We were also able to detect IFN $\alpha$-inducible microRNA genes although the changes observed were moderate and biological significance remains to be proven. Like most microarraybased detection technologies the technical variability among identical samples is low compared to biological variations of individual cell cultures. At this point it is important to note that variation among biological samples occurs and is independent of the parameters that are measured. Consistent with IFN $\alpha$ dependent induction of mRNAs we find that virtually all modulated microRNA genes are upregulated. However, the IFN $\alpha$-induced changes detected in our study are relatively small compared to the changes induced by IFN $\beta$ in $\mathrm{HuH} 7$ cells (9). Finally, it is noteworthy that IRmiRs have similar kinetic properties to their mRNA counterparts. miR-10b for instance is induced early in ME-15 and remains upregulated, while miR-19 abundance ceases after 24 h. In general, the majority of IRmiR genes were reset to basal levels after $24 \mathrm{~h}$ and further studies are needed for kinetic classification. Thus, our study adds another level of complexity to the dynamic regulation IFN $\alpha$ signaling and other mechanisms like epigenetic promoter methylation are currently under intense investigation in our laboratories.

\section{Acknowledgements}

We thank Dr. Guido Steiner and Andreas Buness (F. Hoffmann-La Roche Ltd.) for their support in bioinformatics and statistics, Dr. Martin Ebeling (F. Hoffmann-La Roche Ltd.) for the comparative genomic evaluation of microRNA-targeted transcripts and Prof. Dr. Giulio Spagnoli (University Hospital Basel) for the gift of the ME-15 melanoma cell line. Finally, we are grateful to Heather Hinton (F. Hoffmann-La Roche Ltd.) for critical reading of the manuscript and to Dr. Laura Suter-Dick (F. Hoffmann-La Roche Ltd.) for sharing lab space and introduction into GCP sampling.

\section{References}

1. Borden EC, Sen GC, Uze G, Silverman RH, Ransohoff RM, Foster GR et al (2007) Interferons at age 50: past, current and future impact on biomedicine. Nat Rev Drug Discov 6(12):975-990

2. Certa U, Wilhelm-Seiler M, Foser S, Broger C, Neeb M (2003) Expression modes of interferon-alpha inducible genes in sensitive and resistant human melanoma cells stimulated with regular and pegylated interferonalpha. Gene 315:79-86

3. Zimmerer JM, Lesinski GB, Kondadasula SV, Karpa VI, Lehman A, Raychaudhury A et al (2007) IFN-alpha-induced signal trans- 
duction, gene expression, and antitumor activity of immune effector cells are negatively regulated by suppressor of cytokine signaling proteins. J Immunol 178 (8):4832-4845

4. Yoshimura A, Naka T, Kubo M (2007) SOCS proteins, cytokine signalling and immune regulation. Nat Rev Immunol 7(6):454-465

5. Song MM, Shuai K (1998) The suppressor of cytokine signaling (SOCS) 1 and SOCS3 but not SOCS2 proteins inhibit interferonmediated antiviral and antiproliferative activities. J Biol Chem 273(52):35056-35062

6. Pichiorri F, Suh SS, Ladetto M, Kuehl M, Palumbo T, Drandi D et al (2008) MicroRNAs regulate critical genes associated with multiple myeloma pathogenesis. Proc Natl Acad Sci U S A 105(35):12885-12890

7. Bartel DP (2004) MicroRNAs: genomics, biogenesis, mechanism, and function. Cell 116(2):281-297

8. Friedman RC, Farh KK, Burge CB, Bartel D (2009) Most mammalian mRNAs are conserved targets of microRNAs. Genome Res 19(1):92-105

9. Pedersen IM, Cheng G, Wieland S, Volinia S, Croce CM, Chisari FV et al (2007) Interferon modulation of cellular microRNAs as an antiviral mechanism. Nature 449(7164):919-922

10. Lüscher U, Filgueira L, Juretic A, Zuber M, Lüscher NJ, Heberer M et al (1994) The pattern of cytokine gene expression in freshly excised human metastatic melanoma suggests a state of reversible anergy of tumor-infiltrating lymphocytes. Int J Cancer 57(4):612-619

11. Nakabayashi H, Taketa K, Miyano K, Yamane T, Sato J (1982) Growth of human hepatoma cells lines with differentiated functions in chemically defined medium. Cancer Res 42(9):3858-3863
12. Chen J, Lozach J, Garcia EW, Barnes B, Luo S, Mikoulitch I et al (2008) Highly sensitive and specific microRNA expression profiling using BeadArray technology. Nucleic Acids Res 36(14):e87

13. Bolstad B, Irizarray R, Astrand M, Speed T (2003) A comparison of normalization methods for high density oligonucleotide array data based on bias and variance. Bioinformatics 19:185-193

14. R Development Core Team. R: A language and environment for statistical computing. Vienna, Austria: R Foundation for Statistical Computing 2008. URL: http://www.Rproject.org

15. Du P, Kibbe WA, Lin SM (2008) Lumi: a pipeline for processing Illumina microarray. Bioinformatics 24(13):1547-1548

16. Smyth GK (2005) Limma: linear models for microarray data. In: Gentleman R, Carey V, Dudoit S, Irizarry R, Huber W (eds) Bioinformatics and computational biology solutions using $\mathrm{R}$ and bioconductor. Springer, New York, pp 397-420

17. Benjamini Y, Hochberg Y (1995) Controlling the false discovery rate: a practical and powerful approach to multiple testing. J Roy Stat Soc B Stat Meth 57(1):289300

18. Roush S, Slack FJ (2008) The let-7 family of microRNAs. Trends Cell Biol 18(10): 505-516

19. Gaur A, Jewell DA, Liang Y, Ridzon D, Moore JH, Chen C et al (2007) Characterization of microRNA expression levels and their biological correlates in human cancer cell lines. Cancer Res 67(6):2456-2468

20. Baskerville S, Bartel DP (2005) Microarray profiling of microRNAs reveals frequent coexpression with neighboring miRNAs and host genes. RNA 11(3): 241-247 